\title{
Contents
}

1 A Journey Through Garden Algebras

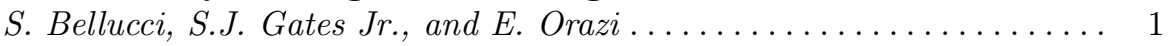

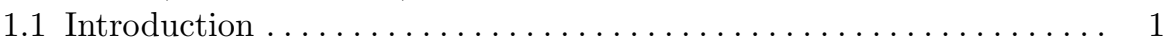

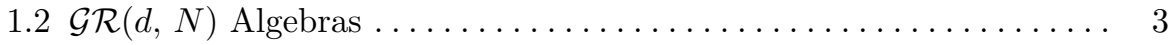

1.2.1 Geometrical Interpretation of $\mathcal{G} \mathcal{R}(d, N)$ Algebras $\ldots \ldots \ldots \ldots$

1.2 .2 Twisted Representations ...................... 6

$1.2 .3 \mathcal{G} \mathcal{R}(d, N)$ Algebras Representation Theory . . . . . . . . . . . 7

1.3 Relationships Between Different Models . . . . . . . . . . . . . . . . 13

1.3.1 Automorphic Duality Transformations ................. 13

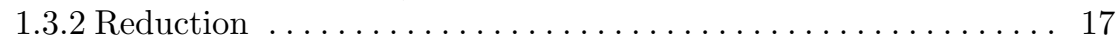

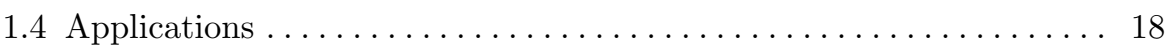

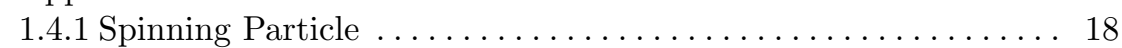

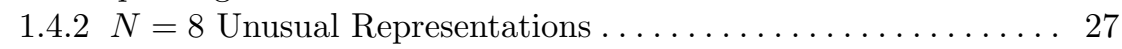

1.5 Graphical Supersymmetric Representation Technique: Adinkras . . . 28

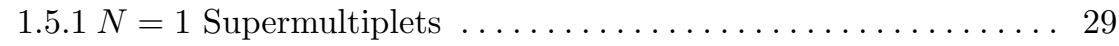

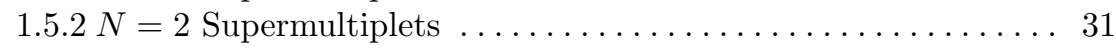

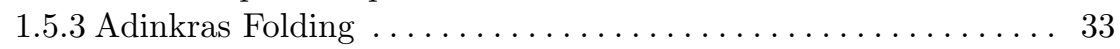

1.5.4 Escheric Supermultiplets . . . . . . . . . . . . . . . . . . . . . 34

1.5.5 Through Higher $N \ldots \ldots \ldots \ldots \ldots \ldots \ldots \ldots \ldots \ldots \ldots$

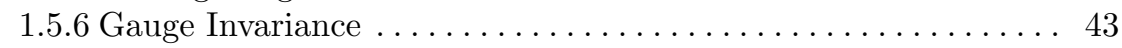

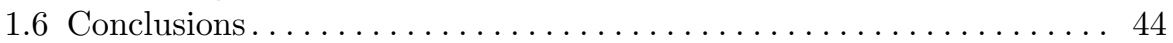

References .............................. 46

\section{Supersymmetric Mechanics in Superspace}

S. Bellucci and S. Krivonos ........................... 49

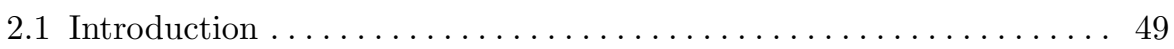

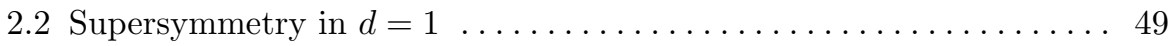

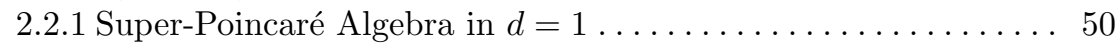

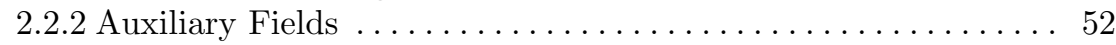

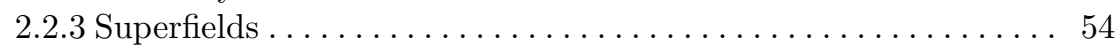

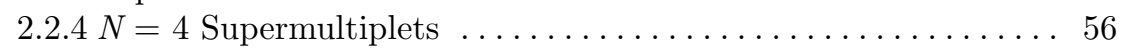




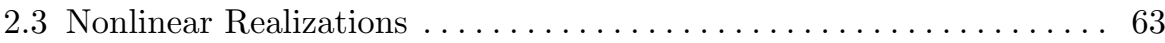

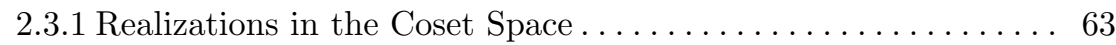

2.3.2 Realizations: Examples and Technique............. 65

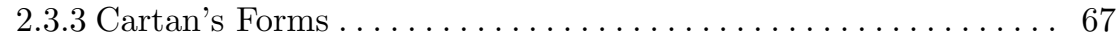

2.3.4 Nonlinear Realizations and Supersymmetry ............. 71

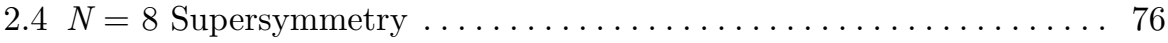

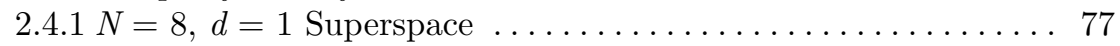

$2.4 .2 N=8, d=1$ Supermultiplets $\ldots \ldots \ldots \ldots \ldots \ldots \ldots \ldots \ldots$

2.4.3 Supermultiplet $(\mathbf{4}, \mathbf{8}, \mathbf{4}) \ldots \ldots \ldots \ldots \ldots \ldots \ldots \ldots \ldots$

2.4.4 Supermultiplet $(\mathbf{5}, \mathbf{8}, \mathbf{3}) \ldots \ldots \ldots \ldots \ldots \ldots \ldots \ldots \ldots \ldots$

2.4.5 Supermultiplet $(\mathbf{6}, \mathbf{8}, \mathbf{2}) \ldots \ldots \ldots \ldots \ldots \ldots \ldots \ldots \ldots \ldots \ldots \ldots$

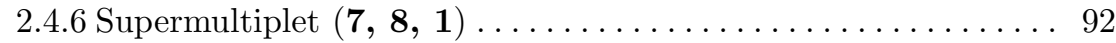

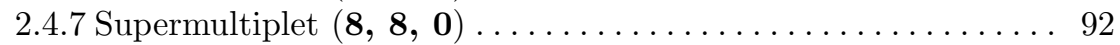

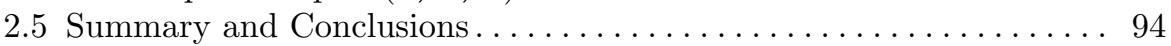

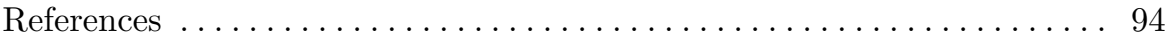

3 Noncommutative Mechanics, Landau Levels, Twistors, and Yang-Mills Amplitudes

V.P. Nair. . . . . . . . . . . . . . . . . . . . . . . . . . . . 97

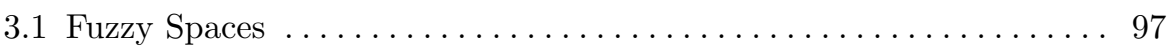

3.1.1 Definition and Construction of $\mathcal{H}_{N} \ldots \ldots \ldots \ldots \ldots 7$

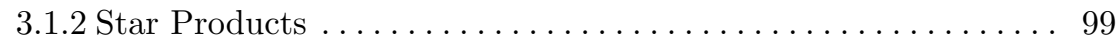

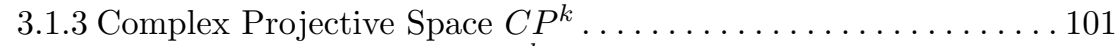

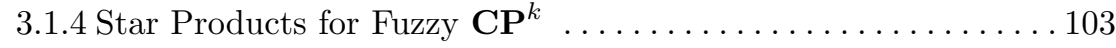

3.1.5 The Large $n$-Limit of Matrices . . . . . . . . . . . . . . . . . . . 105

3.2 Noncommutative Plane, Fuzzy $\mathbf{C P}^{1}, \mathbf{C P}^{2}$, etc. . . . . . . . . . . . . . 107

3.3 Fields on Fuzzy Spaces, Schrödinger Equation. . . . . . . . . . . . . . . 109

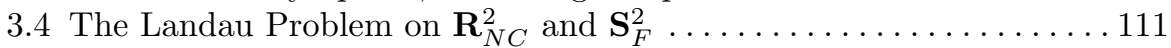

3.5 Lowest Landau Level and Fuzzy Spaces . . . . . . . . . . . . . . 113

3.6 Twistors, Supertwistors . . . . . . . . . . . . . . . . . . . 115

3.6.1 The Basic Idea of Twistors . . . . . . . . . . . . . . . . . . 115

3.6.2 An Explicit Example ............................. 118

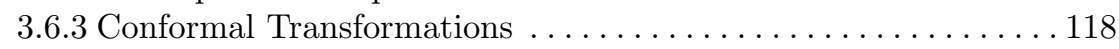

3.6 .4 Supertwistors . . . . . . . . . . . . . . . . . . . . . 119

3.6.5 Lines in Twistor Space . . . . . . . . . . . . . . . . . . . 120

3.7 Yang-Mills Amplitudes and Twistors . . . . . . . . . . . . . 121

3.7.1 Why Twistors Are Useful . . . . . . . . . . . . . . . . . 121

3.7 .2 The MHV Amplitudes . . . . . . . . . . . . . . . . . . . . . . . . 123

3.7.3 Generalization to Other Helicities . . . . . . . . . . . . . . . . 128

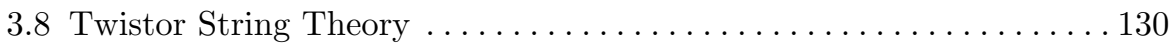

3.9 Landau Levels and Yang-Mills Amplitudes . . . . . . . . . . . . . . . 131

3.9.1 The General Formula for Amplitudes . . . . . . . . . . . . . . . . . 131

3.9.2 A Field Theory on $\mathbf{C P}^{1} \ldots \ldots \ldots \ldots \ldots \ldots \ldots \ldots \ldots \ldots \ldots \ldots \ldots$

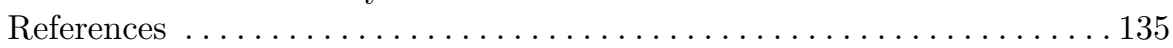


4 Elements of (Super-)Hamiltonian Formalism

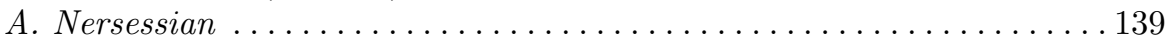

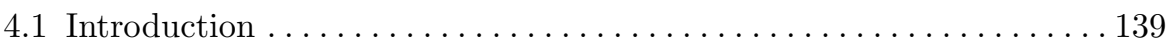

4.2 Hamiltonian Formalism . . . . . . . . . . . . . . . . . . . . . 140

4.2.1 Particle in the Dirac Monopole Field . . . . . . . . . . . . . 143

4.2.2 Kähler Manifolds . . . . . . . . . . . . . . . . . . . . . . . . 145

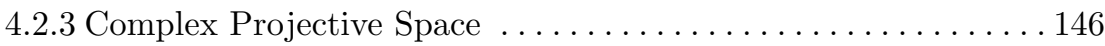

4.2.4 Hopf Maps . . . . . . . . . . . . . . . . . . . . . . . . . . . . . . . . . . . . . . . 147

4.3 Hamiltonian Reduction $\ldots . \ldots \ldots \ldots \ldots \ldots \ldots \ldots \ldots \ldots$

4.3.1 Zero Hopf Map: Magnetic Flux Tube . . . . . . . . . . . . . . 151

4.3.2 1st Hopf Map: Dirac Monopole . . . . . . . . . . . . . . . . . 152

$4.3 .3 \mathbb{C}^{N+1} \rightarrow \mathbb{C P}^{N}$ and $T^{*} \mathbb{C}^{N+1} \rightarrow \mathrm{T}^{*} \mathbb{C P}^{N} \ldots \ldots \ldots \ldots \ldots \ldots \ldots 155$

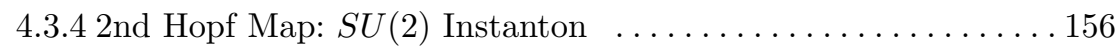

4.4 Generalized Oscillators . . . . . . . . . . . . . . . . . . 160

4.4.1 Relation of the (Pseudo)Spherical Oscillator

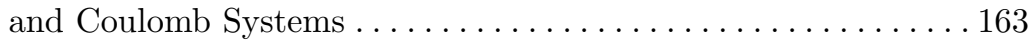

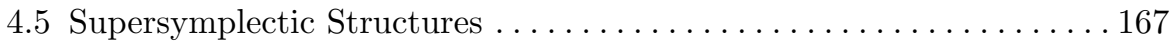

4.5.1 Odd Super-Hamiltonian Mechanics................. 171 4.5.2 Hamiltonian Reduction: $\mathbb{C}^{N+1 . M} \rightarrow \mathbb{C P}^{N . M}, \Lambda \mathbb{C}^{N+1} \rightarrow \Lambda \mathbb{C P}^{N} .172$

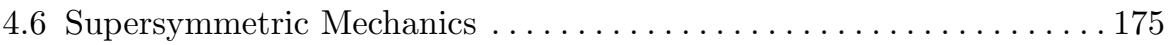
4.6.1 $\mathcal{N}=2$ Supersymmetric Mechanics with Kähler Phase Space. . 177

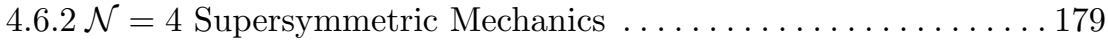

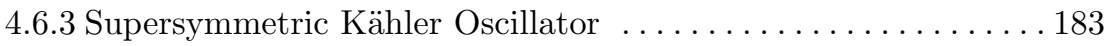

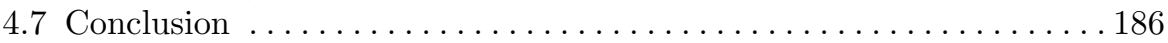

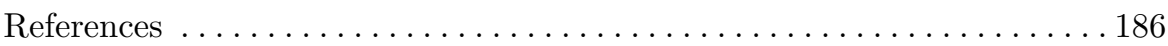

5 Matrix Models

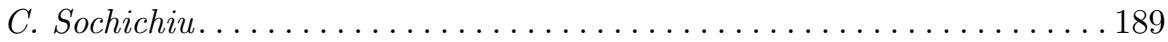

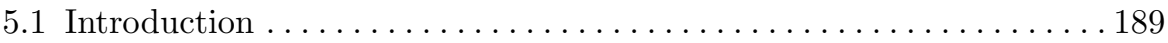

5.2 Matrix Models of String Theory . . . . . . . . . . . . . . . . . 190

5.2 .1 Branes and Matrices ... . . . . . . . . . . . . . . . . . 190

5.2 .2 The IKKT Matrix Model Family . . . . . . . . . . . . . . . 191

5.2 .3 The BFSS Model Family . . . . . . . . . . . . . . . 192

5.3 Matrix Models from the Noncommutativity . . . . . . . . . . . . . 193

5.3.1 Noncommutative String and the IKKT Matrix Model . . . . . . . 193

5.3.2 Noncommutative Membrane and the BFSS Matrix Model . . . . 199

5.4 Equations of Motion: Classical Solutions . . . . . . . . . . . . . 201

5.4.1 Equations of Motion Before Deformation:

Nambu-Goto-Polyakov String . . . . . . . . . . . . . . . . . 201

5.4.2 Equations of Motion After Deformation:

IKKT/BFSS Matrix Models.................... 203

5.5 From the Matrix Theory

to Noncommutative Yang-Mills . . . . . . . . . . . . . . . 206

5.5.1 Zero Commutator Case: Gauge Group of Diffeomorphisms . . . 206 5.5.2 Nonzero Commutator: Noncommutative Yang-Mills Model . . . . 209 


\section{Contents}

5.6 Matrix Models and Dualities

of Noncommutative Gauge Models . . . . . . . . . . . . . . . . . . . . . . . 215

5.6.1 Example 1: The $U(1) \longrightarrow U(n)$ Map $\ldots \ldots \ldots \ldots \ldots \ldots \ldots . \ldots 217$

5.6.2 Example 2: Map Between Different Dimensions . . . . . . . . . . 220

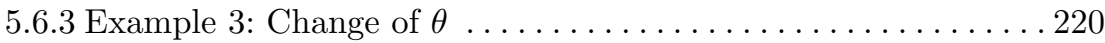

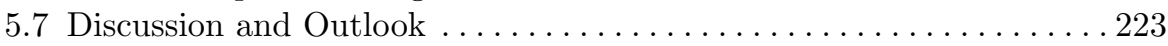

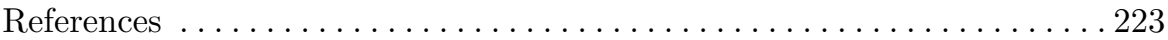

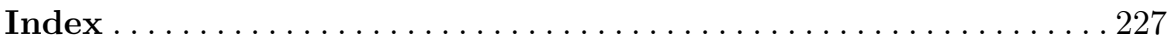

